\title{
143. Electron Microscopic Observation of Intracranial Tumors
}

\author{
Naoki Kageyama, M.D. and Taro Fukumitsu, M.D. \\ First Division of Surgical Department, Kyoto University \\ School of Medicine
}

\section{1) Pituitary adenoma}

Cells of eosinophilic adenoma (one case) contain granules of a general diameter about $300 \mathrm{~m} \mu, 400 \mathrm{~m}_{\mu}$ at maximum, showing nearly the same picture as those of normal alpha cells (diameter of granules 300 to $400 \mathrm{~m} \mu$ ). Cells of basophilic adenoma (one case) are also usually granules of $300 \mathrm{~m} \mu$ in diameter (400 $\mathrm{m} \mu$ at maximum) which are different from normal beta cells (granule diameter is less than $200 \mathrm{~m} \mu$ ). No difference of granule in size was found in these two tumors. It seems, therefore, at least in tumors, the size of granules does not agree with the stainability of them.

In 8 cases of chromophobe adenoma, no chromophilic granule was found even with specific staining techniques when examined under a light microscope; but when examined with an electron microscope, though smaller in number, secretory granules of about 70 to $200 \mathrm{~m} \mu$ in diameter (most of them about $100 \mathrm{~m} \mu$ ) were recognized in nearly all cases.

These granules tend to palisade along the cell membrane, occasionally showing a secretory picture into the cellular interspace. In many cases, granules were found in all cells, but in a few cases, they were contained only in some tumor cells, and it seemed that more granules were found in cells around the blood vessels. Golgi apparatus has been thought to be the site of production of this granule and similar findings supporting this idea were found in our tumor cases.

\section{2) Giant-celled glioblastoma}

There has been much discussion on the tissue origin of this tumor. This is a typical case containing nerve cell-like giant cells, sharply demarcated from brain tissue, without any proliferation of vessel wall and showed the presence of reticuline fiber. The electron microscopical pattern was the same as that of other mesenchymal tumor in which collagenous fibers were closely attached to the cell membrane in most of the tumor cells.

A very peculiar point of this tumor is, it contains cells which have well developed collagenous fibers in the cytoplasms, and the mode of collagenous fiber production is also quite unique. Even in those portions where no collagenous fiber is present, the shape of cells is also unique and they do not appear like glial cells.

From these findings, at least this case is not glioma but a sarcoma. Two other cases showed somewhat similar histological picture in some respect, but no collagenous fiber was found electron microscopically, and many other findings 
with a light microscope suggested a possible glial origin.

Therefore, it is considered that in so-called giant celled glioblastoma, there may be tumors of glial origin, but sarcomas are also, surely included in this group.

\title{
144. On the Total Removal of Craniopharyngioma
}

\author{
J. Suzuki, T. Wada, K. Sawada and S. Saso \\ Katsura Surgical Clinic, Tohoku University
}

Because of its originating position, total removal of craniopharyngioma includes many difficult problems in regard to the operation and postoperative hormonal control and there are only few reports of the operation in the list.

Recently, five cases of craniopharyngioma, one adult and four children, were experienced in Katsura clinic. One of them expired three months after the operation but other four, including in-patients, are leading a good course.

First we refer to operative approach, considerations in deciding the side to operate, and the operation itself.

Next, we state over the usefulness of hypothermic anesthesia on the bases of postoperative behaviors of 17-OHCS and ADS, furthermore over some precautions in administrating cortisone and water in order to control possible diab. insip. and finally over some postoperative functional change of the thyroid gland.

Here, the body-growth rate and changes in sexual function are very interesting problems, which remain to be solved in the future.

\section{Study on Pre- and Post-Operative Pituitary Adrenal Function of Sellar and Parasellar Tumors}

\author{
Kenzo Matsuoka, Akira Kitagawa, Toru Uozumi, Ryoichi NozaKi \\ First Department of Surgery, Osaka University Medical School
}

We already published the study on ACTH-Z Test to confirm the secondary adrenal insufficiency of sellar and parasellar tumors. One case of shock due to adrenal failure in post-operative course was experienced in the cases revealed hyporeactive response.

By the surgical invasion to preoperative hypofunctional cases to remove the pressure due to tumor without giving hypophyseal lesion, rapid recovery of adrenal 